\title{
A Real-Time Energy Management Platform for Multi- vector District Energy Systems
}

\author{
Muhammad Waseem Ahmad ${ }^{1}$, Jonathan Reynolds ${ }^{1}$, Jean-Laurent Hippolyte ${ }^{1}$, \\ Yacine Rezgui ${ }^{1}$, Michael Nikhil Descamps ${ }^{2}$, Christian Merckx ${ }^{3}$, Jasper van Dessel ${ }^{3}$, \\ Mathieu Lessinnes ${ }^{3}$ \\ ${ }^{1}$ BRE Centre for Sustainable Engineering, School of Engineering, Cardiff University, \\ Cardiff, United Kingdom, CF24 3AA. \\ ${ }^{2}$ Département Thermique Biomasse et Hydrogène, Commissariat à l'énergie atomique et \\ aux énergies alternatives, France. \\ ${ }^{3}$ Tractebel Engineering SA, Boulevard Simon Bolivar, 34-36, 1000 Brussels, Belgium. \\ \{AhmadM3, ReynoldsJ8, HippolyteJ, RezguiY\}@cardiff.ac.uk \\ \{Michael.Descamps\}@cea.fr \\ \{christian.merckx, jasper.vandessel, mathieu.lessinnes\}@tractebel.engie.com
}

\begin{abstract}
Management of increasingly complex, multi-vector, district energy systems, operated by separate stakeholders, including prosumers, is a vital challenge to overcome in a fragmented energy landscape. The complex value chain involved forms a cognitive virtual network with the shared objective to reduce energy consumption, greenhouse gas emissions and maximise human comfort. This paper will aim to illustrate the PENTAGON platform for integrated management of key stakeholder data to produce automatic, holistic and pre-emptive decisions that ensure near-optimal management of a district energy system. The PENTAGON platform architecture consists of five key modules including Smart Connector to interface with existing District Energy Management Systems (DEMS), a time series database, a prediction module, a multi-vector optimisation module and a module ensuring the electric grid stability. Integration of these distinct modules is achieved through an underpinning, shared, semantic description of the district components, sensors and scenarios. The ultimate goal of the described platform is to achieve a stepchange from static, reactive, rule-based systems to an intelligent, adaptive, and pre-emptive control architecture that makes new decisions based on perceived and predicted conditions
\end{abstract}

Keywords: District energy management; ICT platform; artificial intelligence; multi-vector energy systems;

\section{Introduction}

The existing building sector, one of the most substantial consumers of energy, contributes towards $40 \%$ of world's total energy consumption and $30 \%$ of the total $\mathrm{CO}_{2}$ emissions [1]. Currently, most of the installed energy systems are based on fossil 
fuels, and more focus on improving energy efficiency, integrating renewable energy sources and optimally managing energy systems is needed to meet national and international obligations. Decentralising energy infrastructure can also increase energy efficiency as it could reduce transmission losses and co-generation or trigeneration units could be utilised. The stochastic nature of renewable energy sources (e.g. wind and solar) increases the complexity in the management of a grid, as they introduce a level of uncertainty into energy supply systems. According to Reynolds et al. [2], the full potential of the smart grid can be utilised by increased interoperability, better energy management and forecasting of both supply and demand. Therefore, it is critical to holistically manage decentralised district energy systems and control a multi-vector energy system in an integrated manner.

Current energy management techniques in district energy systems are limited to relatively simple control mechanisms and don't consider the collaborative aspects of the domain resulting from the multiplicity of stakeholders and physical systems. Advances are needed in developing novel collaborative management and system modelling techniques, and development of management platform for the efficient operation of collaborative networks in energy systems. Mimicking human intelligence in software tools by managing the operation of collaborative networks in energy systems will not only provide better indoor environmental conditions but will also reduce energy consumption. This has enabled the development of complex collaborative systems inspired by human cognition, such as artificial neural networks, fuzzy logic, semantic web technologies, etc.

Our buildings and energy systems are equipped with a large number of heterogeneous devices, which mostly come from different vendors and use different communication protocols. To provide holistic management of energy systems, that are capable of tackling underlying heterogeneity, classification and description of different information within the decentralised energy systems and consumers are needed [3]. Semantic web technologies can improve the interoperability while reducing heterogeneity. These technologies (e.g., ontologies) can facilitate rapid exploration of information, i.e., it becomes easier to retrieve, correlate and integrate data by knowledge discovery, associating meaning to data and providing interrelationships between modelled entities. The proposed energy platform leverages semantic modelling to allow interoperability of different data sources and provides an additional level of robustness to energy management of multi-vector systems. The rest of the paper is organised as follow. Section 2 details energy management platform along with its components; Smart Connector, prediction module, multi-vector optimiser and Smart Operation. In Section 3, we describe ICT architecture and interaction between different modules. Pilot site implementations are discussed in Section 4 and Conclusions are presented at the end of the paper.

\section{PENTAGON Energy Management Platform}

The functional architecture of the PENTAGON multi-vector flexibility management platform is depicted in Fig. 1. The district energy management system (DEMS) gathers measurements and communicates with the PENTAGON platform with the 
help of Smart Connector. In this section, we will describe the functionality of software components of PENTAGON platform along with data flow.

\subsection{Definition of the Platform}

- Smart Connector

Smart Connector acts as a translator, and collects data at every time step from the DEMS and organises it in a predefined structure. This data is then read and treated by other PENTAGON components. The Smart Connector also translates the optimal control actions (i.e., set-points calculated by the multi-vector optimiser) so that they can be interpreted and applied by the DEMS. The Multi-vector optimiser will always send an optimised solution over the next 24 hours, however the Smart Connector will only communicate the next time step (15 minutes) of the solution and will store the remaining $24 \mathrm{~h}$ solutions until the new control actions are available.

- Prediction module

Predictive analytics play an important role in the management of multi-vector energy systems and serve as a core component of smart thermal and electrical grids. The PENTAGON prediction module will manage the prediction models and will forecast different variables. The variables of interest will include thermal and electrical energy demands and energy production from renewable energy sources. To train prediction models, different input features (e.g., outdoor weather conditions, time-related information, building occupancy, etc.) are tested to improve their performance.

- Multi-vector optimiser

Holistic management of decentralised district energy systems is the key to their success. Multi-vector energy networks (e.g., heat, gas and electricity) that were previously independently controlled are now required to be managed and controlled in an integrated manner as they have become more coupled (e.g. output of one systems is the input of second system). The PENTAGON multi-vector optimiser is responsible for computing control actions and set-points to minimise a defined objective function (e.g. minimise energy consumption/cost, increase occupants' comfort, etc.). The optimiser will exploit available flexibility within the district while satisfying the energy demand of the buildings. The optimiser will also consider the specific characteristics of conversion technologies to minimise the overall energy cost of the district while satisfying the systems' and networks' constraints. Internal simplified models will be used to effectively evaluate potential optimal solutions, dispatch, and define operational constraints. The computed power flow and set-point values will then be send to the Smart Operation and District Sever.

- Smart Operation

Smart Operation is a proprietary software tool from Tractebel that is used for the optimisation of an electrical distribution network in the presence of distributed energy resources (DERs) such as photovoltaic (PV) systems, wind turbines, electric vehicles (EVs), battery storage systems, etc. ensuring the adherence to grid constraints. Within PENTAGON, Smart Operation receives the forecasted consumption and production from PENTAGON prediction. It also receives the decisions taken by the 
PENTAGON multi-vector optimiser concerning the electric flexibility and the energy conversions. The later will not be changed in order to preserve the global equilibrium found by the multi-vector optimiser. Only the electric flexibility (state of charge of the batteries, curtailment or shedding) and the withdrawal from/injection to the grid can be re-evaluated by Smart Operation based on a more detailed model of the electric grid. To this aim, a multi-period AC Optimal Power Flow (AC-OPF) is run in which the electrical flows are optimised according to an objective function while respecting the grid constraints, e.g., voltage magnitude and current limits. Smart Operation will keep as much as possible the original dispatch schedule. If deviations are required, they will be minimized while at the same time being different enough to ensure the network security.

\section{IT Architecture and Interaction between PENTAGON Modules}

\subsection{Communication between ICT Components}

In the context of utility control infrastructures, European standardisation bodies recommend service-oriented architecture (SOA) [4], to facilitate the integration of:

- data services;

- functional logic services;

- and business logic services.

In an SOA, software functionalities are packaged in autonomous self-contained modules that operate independently from each other's state and context [5]. Fig. 1 shows the main high-level components of the PENTAGON solution. The diagram points out the Application Programming Interface (API) that each module requires (half circles) and what other module is supposed to provide these interface (full circles). The district optimiser component uses the interfaces provided (1) by PENTAGON model component to retrieve relevant predicted data and to send thermal and gas network related commands, and (2) by the Smart Operation component to send optimised electrical flows.

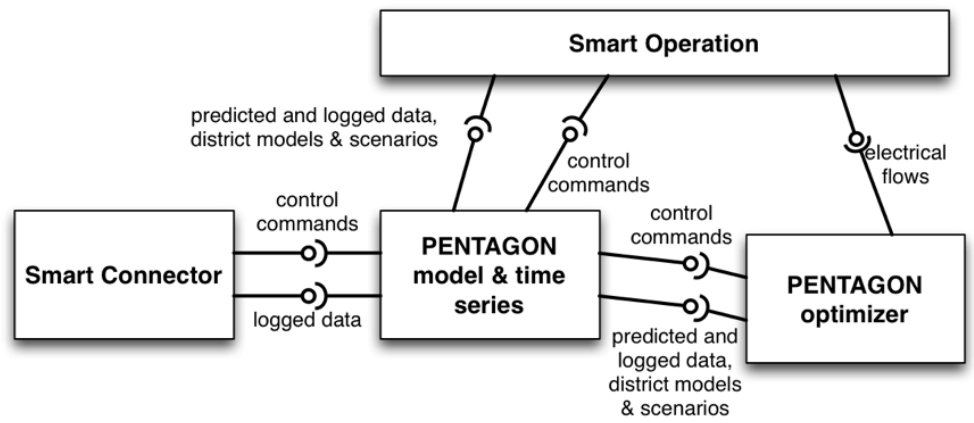

Fig. 1. PENTAGON component diagram 
The Smart Operation component uses the interface provided by the PENTAGON model component to send electrical network related commands, which are derived from the optimised electrical flows received from the district optimization component. The PENTAGON model component uses the interface provided by the district server to fetch the data measured in the district that are required for prediction. SOAs allow for complex yet flexible systems, individual components can evolve independently, assuming changes in interface specifications are kept to a minimum. The most common technique to implement interoperable SOA are web services. The PENTAGON platform has adopted the Representational State Transfer (REST) communicating architecture style. By definition, REST web services are lightweight and stateless [6], which can be regarded as a realisation of the SOA paradigm as it specifies how services are published, discovered, and consumed across the web [6].

\subsection{PENTAGON Data Model}

The PENTAGON data model is made up of different types of information: the topology of the different networks with associated parameters and the configuration parameters of the optimization problem (constraints and objective function). The information is managed by the PENTAGON-model. All the parameters, even those of the nonlinear model of Smart Operation, are part of the PENTAGON data model. None are managed in an external way by a module. Each of the considered test cases is fully represented by a database set and switching from one test case to another is thus automatic. The configuration of the model for each test case is provided in an Excel template, which is then loaded into the database. All parameters of the optimization problem (bounds, limits, prices, objective function type, constraints type, ...) are included in that template. The PENTAGON platform is therefore fully configurable in a very flexible manner. It is worth mentioning that the measurements transferred from the District Server are also registered in the PENTAGON data model.

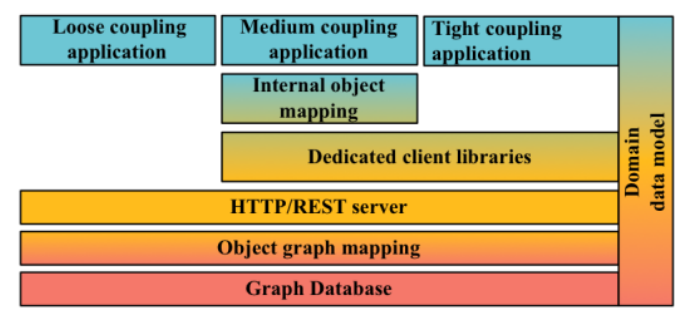

Fig. 2. PENTAGON online transaction processing system and possible degree of coupling

The dynamics of the PENTAGON modules thus rely on an online transaction processing system composed mainly of:

- a database management system that is optimised for retrieval and/or update of semantic data and associated time-dependent data with respect to the complex domain-specific model and;

- the PENTAGON domain data model; 
As shown in Fig. 2, PENTAGON's online transaction processing system drives interoperability with/within the platform, by maintaining consistent semantics throughout the whole PENTAGON stack. This consistency is delivered by systematic mappings between various representations. For instance, JSON objects exposed by the REST API align with the object models of the client libraries through automated code generation and the schema of the underlying (NoSQL) graph database aligns the object models through semi-automated object graph mapping. Modules using the platform (district optimisers, prediction modules, district gateways etc..) can have various degrees of coupling with the PENTAGON data model, from being loosely coupled by forging HTTP requests to retrieve/update data (e.g. a visualisation web application) to being tightly coupled by using the provided PENTAGON object model internally (e.g. the delegate application that connects to the District Server to retrieve time series values).

PENTAGON's domain model aligns and extends previous ontologies proposed by EU projects RESILIENT [7] and MAS2TERING [8]. In RESILIENT, an OWL [9] ontology formalised the socio-technical elements involved in district energy management systems, as well as their interrelationships [10]. The ontology was built on top of existing upper layer abstract ontologies, to achieve a two-fold representation of district energy systems, as systems of systems and as socio-technical networks. In the MAS2TERING [8], an ontology formalized the domain of flexibility management in smart energy systems [11]. The ontology aligns selected standards: the Universal Smart Energy Framework [12], OpenADR [13], Energy@home [14], and CIM [15].

\subsection{Time Series Data Storage}

In recently completed projects, RESILIENT [7] and MAS2TERING [8], the developed ontologies did not consider semantical features to represent time indexed data, observations from measurements and associated metadata. A number of existing semantic models that standardise the description of time series data, especially the data obtained from sensors and meters will be considered. Among these semantic models are;

- SensorML-SensorML is one of the OGC SWE information-encoding standards and provides models and encoding to represent any kind of process in sensors or post-processing systems [16].

- Semantic Sensor Network Ontology - This ontology is used to describe sensors, observation, and related concept. Domain knowledge (e.g., time, location of a sensor, etc.) are described and imported through other ontologies via OWL imports [17].

- $\quad$ SENML (Media Type for Sensor Markup Language) - This media type can be used in protocols (e.g., HTTP or CoAP) by sensors to send or configure measurements [18]. 


\subsection{Security Requirements}

PENTAGON project deals with sensitive data (i.e. information of energy consumption, etc.) and therefore user authentication will be employed to only allow authorised access. All requests to any service will need the client to be authenticated, and will require users to enter their username and password. Communication security will be further enhanced by using the widely used secure communication HTTPS protocol. It consists of communication over HTTP within a connection encrypted by TLS (Transport Layer Security) or SSL (Secure Socket Layer). It is worth mentioning that the approach described here applied only to the communication between PENTAGON components. However, communication between third-party's services/components (e.g., DEMS, public weather services, etc.) and PENTAGON component might have to comply with different security policies (e.g., defined by district IT network administrators).

\section{Project Pilot Sites and Implementation}

PENTAGON proposes to implement a 2 step validation strategy that involves (i) live operations at limited scale on the experimental district heating network of CEA-INES (Chambery, France) and (ii) a wider assessment (low voltage and medium voltage level) of optimization capabilities based on simulations of the Blaenau Gwent district (Wales, UK). The experimental facility of CEA-INES is a scaled heating network with a set of heat generators, thermal storage, a network of pipes and valves, and heat consumers. On the heat production side, the facility includes a $300 \mathrm{~kW}$ gas boiler, a $100 \mathrm{~kW}$ absorption chiller, $300 \mathrm{~m}^{2}$ of solar panels, and $50 \mathrm{~kW}$ heat pump is being deployed in the context of the project. On the consumption side, the energy is distributed to a thermal test rig, which can emulate building energy load in a flexible and reproducible way. This means that a given heat demand can be applied to the network, and heat production control variables can be tuned to satisfy the demand. In addition, thermal flexibility takes the form of a $40 \mathrm{~m}^{3}$ water storage tank. The facility is managed and monitored by an instrumentation server that collects all data coming from sensors and actuators. The heat production units can be run in parallel or separately. The thermal storage provides flexibility to the system, and can be bypassed if required. A set of actuator valves are activated to choose between the different heat production units or to bypass the thermal storage. The building load is applied to the consumer heat exchanger using the building load profiles provided by PENTAGON-simulation.

The validation by simulations of the Blaenau Gwent district requires a heating network simulator and an electric network simulator. The heating network is simulated using the equation-based object-oriented language Modelica along with the simulation platform Dymola. The dynamic heating network model is based on detailed physical representation of the system by gathering component models that were previously developed and validated. The components models are taken from a CEA in-house Modelica library named DistrictHeating [19]. At the production plant 
level, the model enables the control of supply temperature and differential pressure, and takes into account heat propagation delays, heat losses, tube thermal inertia, and pressure losses. At the consumer level, the buildings are not explicitly modelled, but represented by their substation, which is the unit in which the energy is distributed from a high level to a low level. The substation is modelled as a system which includes a heat exchanger, a regulation valve, and an ideal controller. With this representation, building heat load temporal profiles can be used as inputs to the model. The electric network simulator is a multi-period load flow calculator which needs the power consumption and production of the different buildings and equipment. Those will be predefined based on historical measurements. The consumer side of the district (electric and heating energy consumption) will be modelled by using computational intelligence techniques (e.g., artificial neural networks, support vector machines, random forest, etc.). The models are capable of simulating complex building energy behaviour due to seasonal variations in weather conditions as well as system non-linearities and delays. The inputs of the models will be weather conditions, time related information, building occupancy, and past values of the variable.

\section{Conclusions}

This paper has introduced the PENTAGON platform for intelligent management of multi-vector energy networks. The platform consists of a collaborative network of modules coordinated through a shared, semantic understanding of the given energy networks. Vitally, the platform allows a paradigm shift from reactive, rule-based control to predictive, anticipatory control and management to attain energy and emissions reductions and maximise human comfort. This is achieved through a combination of real-time data retrieval and organisation through the smart connector, prediction of pivotal variables through leveraging artificial intelligence, deployment of advanced optimisation techniques and adjustment to real-time grid constraints though simulation all underpinned by a central, coordinating ICT infrastructure. Current energy management systems do not consider the collaborative aspects of the domain; PENTAGON platform uses semantic modelling to describe the district energy system to ensure interoperability and communication between ICT modules. Secure communication, one of the key element for real-time management of smart energy systems, will also be implemented as part of PENTAGON platform. The presented platform will be deployed and tested at two eco-districts.

\section{References}

1. Ahmad, M.W., Mourshed, M., Mundow, D., Sisinni, M., Rezgui, Y.: Building energy metering and environmental monitoring - A state-of-the-art review and directions for future research. Energy and Buildings, 120, pp: 85-102 (2016) 
2. Reynolds, J., Ahmad, M.W., Rezgui, Y.: Holistic Modelling Techniques for the operation optimisation of multi-vector energy systems. Energy and Buildings, 131 (2018), doi: https://doi.org/10.1016/j.enbuild.2018.03.065

3. Tomašević, N.M., Batić, M.Č., Blanes, L.M., Keane, M.M., Vraneš, S.: Ontologybased facility data model for energy management. Advanced Engineering Informatics, 29(4), pp: 971-984 (2015)

4. CEN/CENELEC/ETSI.: Joint Working Group on Standards for Smart Grids, Final report (2011)

5. Papazoglou, M.P., W.-J. v. d. Heuvel, W.-J.v.d.: Service oriented architectures: approaches, technologies and research issues. International Journal on Very Large Databases, 16, pp: 389—415 (2007)

6. Sheng, Q.Z., Qiao, X., Vasilakos, A.V., Szabo, C., Bourne, S., Xu, X.: Web services composition: A decade's overview. Information Sciences, 280, pp: $218-238$ (2014)

7. CORDIS.: Coupling Renewable, Storage and ICTs, for low carbon intelligent energy management at district level. Publications Office of the European Union, http://cordis.europa.eu/project/rcn/104392 en.html (2017)

8. CORDIS.: Multi-Agent Systems and Secured coupling of telecom and energy grids for next generation smartgrid services. Publications Office of the European Union, http://cordis.europa.eu/project/ren/192066 en.html (2017)

9. OWL Working Group.: OWL - Semantic Web Standards. Semantic Web Standards, https://www.w3.org/2001/sw/wiki/OWL (2013)

10. RESILIENT consortium.: D2.4 - Architecture description of the information model for district energy management (2014)

11. Hippolyte, J.L., Howell, S., Yuce, B., Mourshed, M., Sleiman, H.A., Vinyals, M., Vanhee, L.: Ontology-based demand-side flexibility management in smart grids using a multi-agent system. In: IEEE International Smart Cities Conference (ISC2), Torento, Italy, pp: $1-7$ (2016)

12. USEF.: Usef Energy - Universal Smart Energy Framework. https://www.usef.energy/ (2017)

13. OpenADR Alliance.: OpenADR - Control Your Energy Future. http://www.openadr.org/ (2017)

14. [36] Energy@Home.: Energy@Home - Home. http://www.energyhome.it/SitePages/Home.aspx (2017)

15. IEC.: Energy management system application program interface (EMS-API) Part 301: Common information model (CIM) base (2011)

16. Nengcheng, C., Hu, C., Chen, Y., Wang, C., Gong, J.: Using SensorML to construct a geoprocessing e-Science workflow model under a sensor web environment. Computers \& Geosciences, 47, pp: 119-129 (2012)

17. Semantic Sensor Network Incubator Group.: Semantic Sensor Net Ontology. [Online].

Available: https://www.w3.org/2005/Incubator/ssn/wiki/Semantic Sensor Net Ontology. [Accessed 3105 2017] (2011)

18. Jennings, C., Shelby, Z., Arkko, J.: Media Types for Sensor Markup Language (SENML). IETF Trust, https://tools.ietf.org/html/draft-jennings-senml-08 (2012) 
19. Giraud, L., Bavière, R., Vallée, M., Paulus, C.: Presentation, validation and application of the DistrictHeating Modelica library. In: Proceedings of the $11^{\text {th }}$ Int. Modelica Conference, Versailles, France, pp: 79-88 (2015) 